\title{
Analysis of the Influencing Factors on the Load-deformation Skeleton Curves of PDCB
}

\author{
Li Ming \\ Civil EngineeringCollege \\ ShenyangJianzhuUniversity \\ Shenyang, China \\ Jangho Group Co., Ltd. \\ Beijing, China \\ e-mail: dingxianxing@126.com
}

\author{
Zhang Haiyang \\ Civil Engineering College \\ Shenyang Jianzhu University \\ Shenyang, China \\ e-mail: 349753653@qq.com
}

Wang Yuanqing

Civil EngineeringCollege

Tsinghua University

Beijing, China

e-mail: wang-yq@mail.tsinghua.edu.cn

\author{
Tao Wei \\ Jangho Group Co., Ltd. \\ Beijing, China \\ e-mail: taowei@jangho.com \\ Wang Bin \\ Jangho Group Co., Ltd. \\ Beijing, China \\ e-mail: wangbin06@jangho.com
}

Shan Renliang

Civil Engineering and Mechanics

China University of Mining\&Technology

Beijing, China

e-mail: srl@cumtb.edu.cn

\begin{abstract}
In the frame-shear wall structure (FSWS) of reinforced concrete, the coupling beam ofshear wall(CB)and frame beam $(\mathrm{FB})$ up the corresponding part of which has a stage of workingcooperatively under horizontal load.However,the research on the whole working mechanism of the $\mathrm{CB}$ and $\mathrm{FB}$ under that stage is little.By taking parallel double coupling beam(PDCB) with different width (PDCBDW) which is constituted of the $\mathrm{CB}$ and $\mathrm{FB}$ as research object, the loading process of 11PDCBare simulated under low-cycle repeated load by nonlinear finite element software. The results show that: with the increase of span-depth ratio of the $F B$, the yield $\operatorname{load}(Y L)$ andultimateload(UL)decreases, the yield displacement(YD), ultimate displacement(UD) and ductility factor(DF) increases. With the decrease of span-depth ratio of the CB, it presents rebound phenomenain skeleton curve. With the increase of the section width of the FB,the YL, UL and DF increase. With the increase of concrete strength, the YL and UL increases. With the increase of the reinforced area ratio, the YL and UL increase. This implies that the span-depth ratiois an important factorwhich influences the skeleton curve.
\end{abstract}

Keywords-Double Coupling Beams; Load-deformation;

skeleton curve; influencing factor; yield displacement

\section{INTRODUCTION}

In reinforced concrete frame-shear wall structure (FSWS), there are parallel double coupling beams (PDCB) with different size, which is composed of part of frame beam (FB) and coupling beam (CB)of shear wall in FSWS. In shear wall structure, there are also PDCB, which is composed of two coupling beams with the same width.
But these two kinds of PDCB are different not only because the width of the beams is different, but also because the reinforcement requirement of them is also different[1]. Many different kinds of CB are promoted by different researchers through changing reinforcement arrangement, taking new structure measurement or changing materialetc, and much research work is also done on them accordingly[2-5]. But little study is done on the PDCB in FSWS. Therefore, it becomes one of the focuses of civil engineers on how the force behavior of parallel double coupling beams with different widthis [6]. So, in this paper, thefactors which influenced the skeleton curveof the load-displacement will be analyzed.

\section{DESIGNED SPECIMENS}

The factors that influence the mechanical properties of PDCB mainly includes the span length(SL) of the CB and the $\mathrm{FB}$, the section height $(\mathrm{SH})$, the section thickness(ST), the span-depth ratio(SDR) of the beams, the concrete strength(CS) and the reinforced area ratio(RAR) which is the area ratio of longitudinal reinforcement in the $\mathrm{CB}$ and the FB. In order to analysis the influence on theskeleton curvesof load-deformation(SCLD) of these factors, 11 specimensare designedshown in Table1. The longitudinal tensile bar of the $\mathrm{CB}$ and the FBare all HRB335, at the same time, the stirrup of them are all HPB300. 
TABLE I. LIST OF THE SPECIMENS

\begin{tabular}{|c|c|c|c|c|c|c|c|c|c|c|}
\hline \multirow[b]{2}{*}{ No } & \multicolumn{4}{|c|}{ FB } & \multicolumn{4}{|c|}{$\mathrm{CB}$} & \multirow[b]{2}{*}{ CS } & \multirow[b]{2}{*}{ RAR } \\
\hline & $\begin{array}{c}\mathrm{SL} \\
(\mathrm{mm}) \\
\end{array}$ & $\begin{array}{c}\mathrm{SH} \\
(\mathrm{mm}) \\
\end{array}$ & $\begin{array}{c}\text { ST } \\
(\mathrm{mm}) \\
\end{array}$ & SDR & $\begin{array}{c}\mathrm{SL} \\
(\mathrm{mm}) \\
\end{array}$ & $\begin{array}{c}\mathrm{SH} \\
(\mathrm{mm}) \\
\end{array}$ & $\begin{array}{c}\mathrm{ST} \\
(\mathrm{mm})\end{array}$ & SDR & & \\
\hline 1 & 1200 & 800 & 300 & 1.5 & 1200 & 400 & 200 & 3 & 14.3 & 2 \\
\hline 3 & 1200 & 600 & 300 & 2 & 1200 & 400 & 200 & 3 & 14.3 & 2 \\
\hline 4 & 1200 & 600 & 300 & 2 & 1200 & 800 & 200 & 1.5 & 14.3 & 2 \\
\hline 6 & 1200 & 600 & 400 & 2 & 1200 & 400 & 200 & 3 & 14.3 & 2 \\
\hline 7 & 1200 & 600 & 250 & 2 & 1200 & 400 & 200 & 3 & 14.3 & 2 \\
\hline 8 & 1200 & 480 & 300 & 2.5 & 1200 & 400 & 200 & 3 & $\mathrm{C} 25$ & 2 \\
\hline 9 & 1200 & 480 & 300 & 2.5 & 1200 & 400 & 200 & 3 & C35 & 2 \\
\hline
\end{tabular}

\section{FINITE ELEMENT SIMULATION METHOD}

In order to simulating the load-bearing process of PDCB, blocksare set at both of the beam ends to make it easy to apply low cyclic load, shown as Fig .1. The loading process of the PDCB is simulated by finite element software.In simulation: the stress-strain curve of the concrete is plastic damage model;eight-node reduced integration of the three-dimensional solid elements(C3D8R)are used to simulate the concrete and upper and lower blocks. TRUSS elements are used to simulate steel. The "embedded" is used to simulate the contact between steel and concrete [7-10]. The lower block is consolidated by limiting displacement of all-directions. The vertical displacement and internal and external plane rotation of the upper block is limited, which makes it can solid along horizontal direction.Ant-symmetric low cyclic loading is applied on both ends of the upper block.Newton-Raphson iteration methodis adoptedto solve the problem.The finite element model is shown as Fig .2.
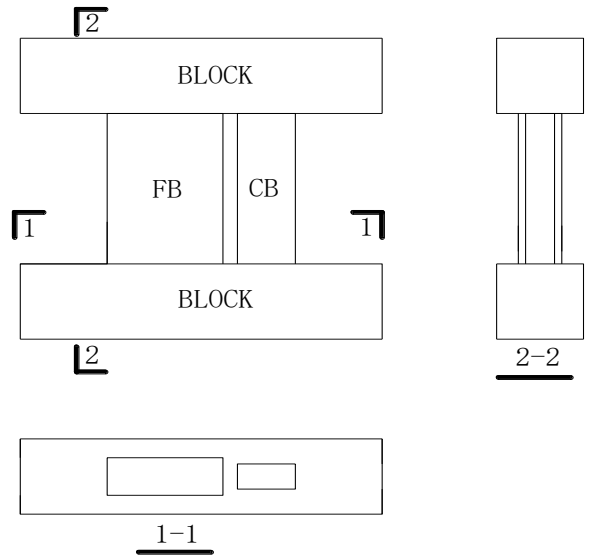

Figure 1. designed PDCB

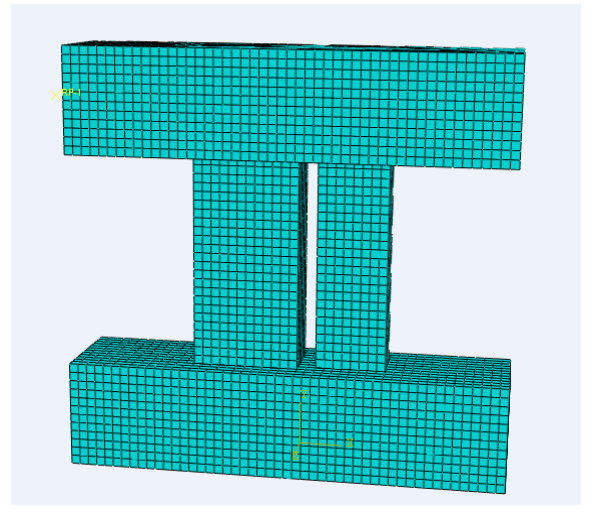

Figure 2. Finite element model of PDCB

\section{ANALYSIS OF INFLUENCING FACTORS ON LOAD-DEFORMATION SKELETON CURVES}

In order to analyze the factors that influence the PDCB,the skeleton curvesof specimen arecalculatedand the key parameters such as the yield load, theultimateloadand ductility factor are also calculated according "The General Yield Moment Method"[11-13]. The skeleton curves are shown as Fig .3 toFig .7.

\section{A. Influence of the span-depth ratiod}

The skeleton curves of specimensnumbered 1,2and3 are shown in Fig .3, of which thespan-depth ratio of the FB is changed.It can be seen from the figures, when the span-depth ratio changes from 1.5 to 2.5 , the yield load(YL) and the ultimate load(UL) all decreases by about $47 \%$, the yield displacement(YD)and the ultimate displacement(UD) increases by about $18 \%$ and $22 \%$ respectively, the ductility factor increases $5 \%$. It shows that with the increase of span-depth ratio of the frame beams, the YL and the UL decreases, the YD, the UD and ductility factor increases. 


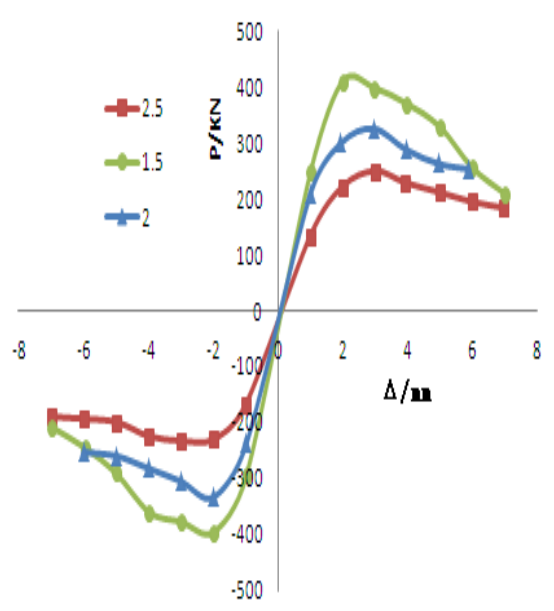

Figure 3. The influence of $\alpha$ totheP- $\Delta$ Skeleton curves

\section{B. Influence of span-depth ratio $\beta$}

The skeleton curves of specimensnumbered 3,4and5 are shown in Fig .4,of which thespan-depth ratio of the $\mathrm{CB}$ is changed.It can be seen from the figures, when the span-depth ratio changes from 1.5 to 3 , the YL increases by about $5 \%$ and the ULincreases by about $18 \%$, the YDdecreases by about $15 \%$ and the UD increases by about $23 \%$ after the first decrease by about $20 \%$, the ductility factorincreases by about $32 \%$ after the first decrease by about $26.6 \%$.From this we can conclude that with the decrease of span-depth ratio of the $\mathrm{CB}$, the YL and UL increases, the YD decreases, the UD and the ductility factor increases after the first reduction, which exhibits rebound phenomena on skeleton curve.

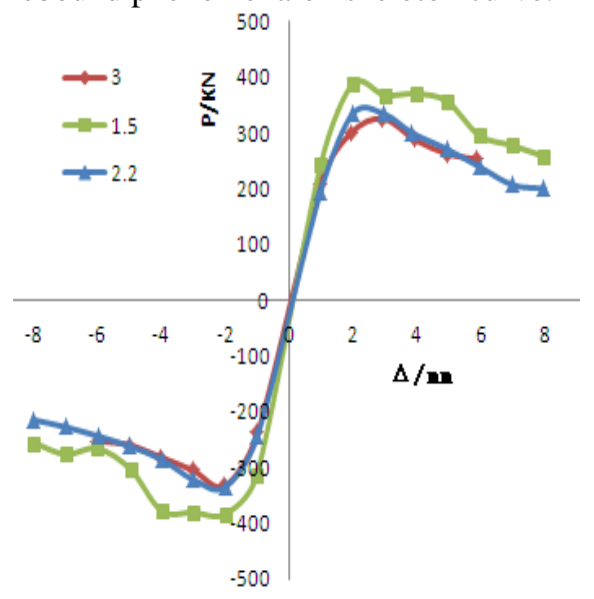

Figure 4. The influence of $\beta$ totheP- $\Delta$ Skeletoncurves

\section{Influence of the section width $B$}

The skeleton curves of specimensnumbered 6,3and7 are shown in Fig .5, of which thesectionwidth of the FB is changed.It can be seen from the figures, when the sectionwidth changes from 250 to 400 , the $\mathrm{YL}$ and the UL decreases by about $3 \%$ and $6 \%$ respectively, the YD and the UD decreases by about 10\% and 33\% respectively, the ductility factordecreases by $33 \%$. It shows that with the increase of the section width of the FB, the YL, the UL and the ductility factor increases, the YD and the UD decreases.

\section{Influence of concrete strength $f_{c k}$}

The skeleton curves of specimensnumbered 8,10 and 9 are shown in Fig .6, of which theconcrete strength is changed.It can be seen from the figures, when the concrete strengthchanges from 25 to 35 , the YL and the UL all increases by about $12 \%$ of, the YDincreases by about $9 \%$,the UD decreases by about $6 \%$ after the first increase by about $9 \%$, the ductility factor decreases by about $14 \%$ after the first increase by about $11 \%$. It shows that with the increase of the concrete strength,theYL and UL increases, the UD and ductility factor decreases after the first increase.

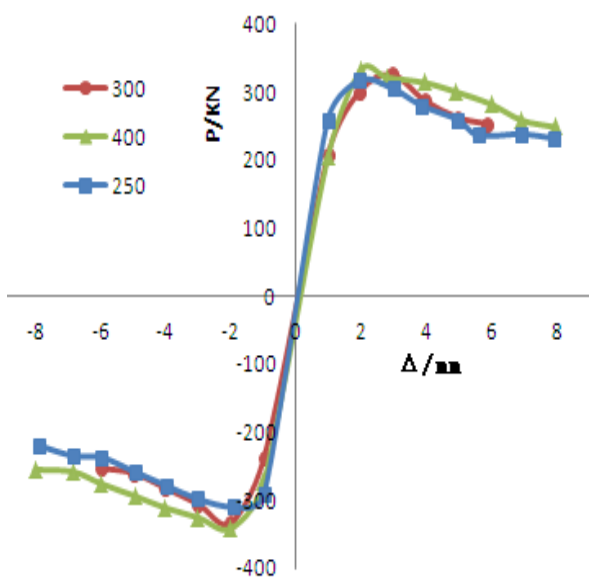

Figure 5. The influence of B totheP- $\Delta$ Skeleton curves

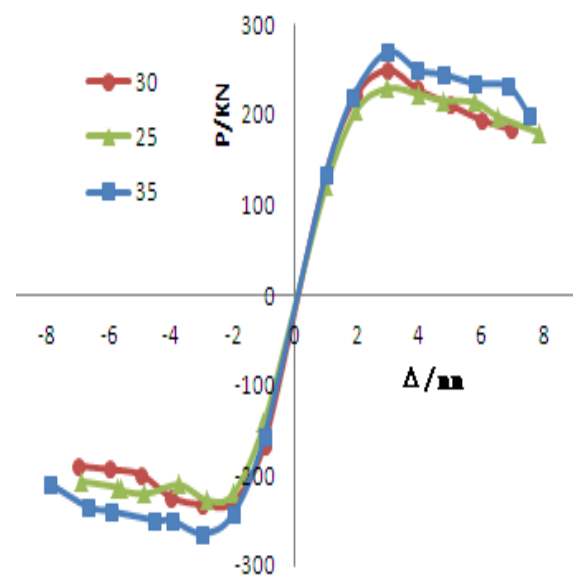

Figure 6. The influence of fckto Skeleton curves

\section{E. Influence of reinforced area ratios.}

The skeleton curves of specimensnumbered 11,2 and 10 are shown in Fig .7, of which theconcrete strength is changed.It can be seen from the figures, when the reinforced area ratiochanges from 1.5 to 2.5 , the $\mathrm{YL}$ and the UL all decreases by about10\%, the YD is almost unchanged, the UD andthe ductility factor all increases by about $15 \%$, and in the descending stage,the bearing capacity reduces slowly with the increase of the reinforced 
area ratio. It shows that with the increase of the reinforced area ratio, the YL and the UL has all increases, the YDis almost unchanged, the UD decreases and the ductility factor reduces.

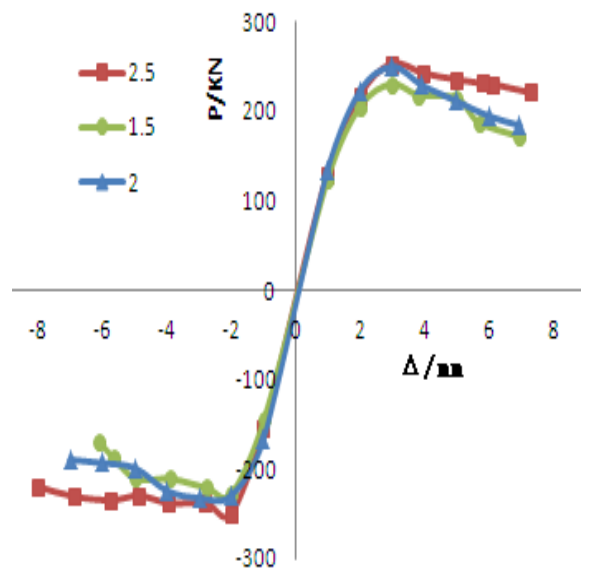

Figure 7. The influence of sto Skeleton curves

\section{SUMMARY}

Based on the above analysis, the following conclusions can be drawn:

With the increase of span-depth ratio of the $\mathrm{FB}$, the YL and UL decreases, the YD, the UD and ductility factor increases.With the decrease of span-depth ratio of the $\mathrm{CB}$, the YL and UL has increases, the YD decreases, the UD and ductility factor increases after the first reduction.

With the increase of the section width of the FB, the YL, UL and ductility factor increases, the YD and the UD decreases.

With the increase of the concrete strength, the YL, UL increases, the UD and ductility factor decreases after the first increase. With the increase of the reinforced area ratio,the YL and the UL all increases, the YDis almost unchanged, the UDand the ductility factor decreases.

\section{ACKNOWLEDGEMENT}

This work was financially supported by China Postdoctoral Science Foundation funded project and Beijing Postdoctoral Research Foundation

\section{REFERENCES}

[1] Cui Jingjing. "Seismicperformanceanalysis of frame-shear wall structure,'DalianUniversityOfTechnology,2012.

[2] CaoWanlin, ZhangJianwei, YinHaipeng, ChenJialong."Experimental study on the seismic behavior of recycle concrete frame-shear-wall structure ,'Engineer Mechanics, 2010,vol.27,pp.135-141.

[3] Shuai Jin."Experimental study on the seismic behavior of the frame-shear-wall structure of steel tube concrete,"Southwest Jiaotong University,2011

[4] Zhou Jinghai , Wang Zhize,Meng Xianhong,Yang Yongsheng."Influence of Aspects Ratios on Seismic Behavior ofRecycled Concrete Lattice Walls, ’Journal of Shenyang Jianzhu University,2012, vol. 28, pp. 241-248.

[5] Jia Lianguang,Iang Yong, Liu Xin,Chen Yong,Zhang Zhuangnan.Experimental Study on Joints of Built-in Steel PlateReinforced Concrete Coupling Beam and Shear Wall,'Journal of Shenyang Jianzhu University ,2013, vol. 29 ,pp. 446-453.

[6] Paulay T, Priestley M J N. "Seismic design of reinforced concrete and masonry buildings,"New York: JohnWiley\&Son, 1999.

[7] [7] Li Ming, Chen Jiguang, Zhao Wei Jian, "Research Progress on Coupling Beam,” ICDMA,2013,pp.1156-1159.

[8] [8] MingHui. "Experimental research on seismic behavior of small-span-to-depth-ratiodual coupling beams with HRB500 bar, ,"Chongqing University, 2012.

[9] [9] Liu Jiabin, Chen Yungang, GuoZhengxing Zhang Jianxi. "Test on seismic performance of precast concrete shear wall with U-shaped closed reinforcements connected in horizontal joints ,'Journal ofSoutheastUniversity,2013,vol.43, pp.565-570.

[10] [10] ZhuZhangfeng,GuoZhengxing. "Test on fabricated short shear wall under low cyclic loading," Engineering Mechanics, 2013,vol.30,pp.125-130.

[11] [11]ZhangBinbin,BaiShaoliang,FuJianping,DengLiluo.

"Experimental research of reasonable reinforcementof short coupling beams of shear walls,"Building Science,2005, vol.21, pp.10-15.

[12] [12] Dai Fengtao. "On the calculation of reinforced concrete beam and pile cracking moment and yield moment "Shanxi Architecture,2006, vol.32, pp.79-80.

[13] [13] JiaYigang,TuXuqing. "A non-linear analysis method of moment modulating of continuous RC beams ," Sichuan Building Science,2005, vol.31, pp.46-49. 